\title{
PSMA-11-Derived Dual-Labeled PSMA Inhibitors for Preoperative PET Imaging and Precise Fluorescence-Guided Surgery of Prostate Cancer
}

\author{
Ann-Christin Baranski ${ }^{1}$, Martin Schäfer ${ }^{1}$, Ulrike Bauder-Wüst ${ }^{1}$, Mareike Roscher ${ }^{1}$, Jana Schmidt ${ }^{1}$, Esther Stenau ${ }^{2}$, \\ Tobias Simpfendörfer ${ }^{3}$, Dogu Teber ${ }^{3}$, Lena Maier-Hein ${ }^{2}$, Boris Hadaschik ${ }^{4}$, Uwe Haberkorn ${ }^{5,6}$, Matthias Eder*7,8, \\ and Klaus Kopka*1,8 \\ ${ }^{1}$ Division of Radiopharmaceutical Chemistry, German Cancer Research Center (DKFZ), Heidelberg, Germany; ${ }^{2}$ Division of \\ Computer-Assisted Medical Interventions (CAMI), German Cancer Research Center (DKFZ), Heidelberg, Germany; ${ }^{3}$ Department \\ of Urology, Heidelberg University Hospital, Heidelberg, Germany; ${ }^{4}$ Department of Urology, University Hospital Essen, Essen, \\ Germany; ${ }^{5}$ Department of Nuclear Medicine, Heidelberg University Hospital, Heidelberg, Germany; ${ }^{6}$ Clinical Cooperation Unit \\ Nuclear Medicine, German Cancer Research Center (DKFZ), Heidelberg, Germany; ${ }^{7}$ Division of Radiopharmaceutical Development, \\ German Cancer Consortium (DKTK), partner site Freiburg, and Department of Nuclear Medicine, University Medical Center, \\ Freiburg, Germany; and ${ }^{8}$ German Cancer Consortium (DKTK), German Cancer Research Center (DKFZ), Heidelberg, Germany
}

Resection of tumors using targeted dual-modality probes combining preoperative imaging with intraoperative guidance is of high clinical relevance and might considerably affect the outcome of prostate cancer therapy. This work aimed at the development of dual-labeled prostatespecific membrane antigen (PSMA) inhibitors derived from the established $N, N^{\prime}$-bis[2-hydroxy-5-(carboxyethyl)benzyl] ethylenediamine- $N$, $N^{\prime}$-diacetic acid (HBED-CC)-based PET tracer ${ }^{68}$ Ga-Glu-urea-Lys (Ahx)-HBED-CC ( $\left.{ }^{68} \mathrm{Ga}-\mathrm{PSMA}-11\right)$ to allow accurate intraoperative detection of PSMA-positive tumors. Methods: A series of novel PSMA-targeting fluorescent dye conjugates of Glu-urea-Lys-HBED$\mathrm{CC}$ was synthesized, and their biologic properties were determined in cell-based assays and confocal microscopy. As a preclinical proof of concept, specific tumor uptake, pharmacokinetics, and feasibility for intraoperative fluorescence guidance were investigated in tumor-bearing mice and healthy pigs. Results: The designed dual-labeled PSMA inhibitors exhibited high binding affinity and PSMA-specific effective internalization. Conjugation of fluorescein isothiocyanate $(10.86 \pm 0.94$ percentage injected dose $[\% I D] / g)$, IRDye800CW (13.66 $\pm 3.73 \% \mathrm{ID} / \mathrm{g})$, and DyLight800 (15.62 $\pm 5.52 \% \mathrm{ID} / \mathrm{g})$ resulted in a significantly increased specific tumor uptake, whereas ${ }^{68} \mathrm{Ga}$-Glu-urea-LysHBED-CC-AlexaFluor488 (9.12 $\pm 5.47 \% \mathrm{ID} / \mathrm{g})$ revealed a tumor uptake similar to that of ${ }^{68} \mathrm{Ga}-\mathrm{PSMA}-11(4.89 \pm 1.34 \% \mathrm{ID} / \mathrm{g})$. The first proof-of-concept studies with the clinically relevant candidate ${ }^{68} \mathrm{Ga}$-Glu-urea-Lys-HBED-CC-IRDye800CW reinforced a fast, specific enrichment in PSMA-positive tumors, with rapid background clearance. With regard to intraoperative navigation, a specific fluorescence signal was detected in PSMA-expressing tissue. Conclusion: This study demonstrated that PSMA-11derived dual-labeled dye conjugates are feasible for providing PSMA-specific pre-, intra-, and postoperative detection of prostate cancer lesions and have high potential for future clinical translation.

Received Aug. 27, 2017; revision accepted Nov. 13, 2017.

For correspondence or reprints contact: Ann-Christin Baranski, Division of Radiopharmaceutical Chemistry, German Cancer Research Center (DKFZ), Im Neuenheimer Feld 280, 69120 Heidelberg, Germany.

E-mail: a.baranski@dkfz.de

${ }^{*}$ Contributed equally to this work.

Published online Nov. 30, 2017.

COPYRIGHT (C 2018 by the Society of Nuclear Medicine and Molecular Imaging.
Key Words: fluorescence-guided surgery; PET imaging; prostate cancer; PSMA-11

J Nucl Med 2018; 59:639-645

DOI: 10.2967/jnumed.117.201293

$\mathbf{P}$ importance for the treatment outcome and survival of patients. In prostate cancer, tumor visualization by multiparametric MRI or hybrid imaging has improved significantly over the past few years (1). Despite the excellent preoperative localization of tumors with diagnostic radiopharmaceuticals, the entirely surgical resection of tumor tissue remains challenging $(2,3)$. Hence, surgical intervention for prostate cancer comes with the risk that tumor tissue may be left behind, thereby increasing the potential for cancer recurrence (4). As a result, there is still a huge demand to improve the intraoperative navigation of the surgeon by further simplifying discrimination between malignant and healthy tissue.

Besides radioguided navigation, intraoperative near-infrared imaging has made promising advances to improve the care of prostate cancer patients $(5,6)$. The nontargeting agents methylene blue and indocyanine green represent the first Food and Drug Administration-approved compounds with near-infrared fluorescence properties for clinical use. However, combining radionuclide and near-infrared imaging in dual-modality agents has been proved to merge the strength of both techniques (7). The application of dual-modality indocyanine green- ${ }^{99 \mathrm{~m}} \mathrm{Tc}$-nanocolloids was one of the first approaches emphasizing improved surgical accuracy through detection of sentinel lymph nodes with combined radioactive and fluorescent signals in clinical proof-ofconcept studies (8).

However, the ability of nontargeted imaging to visualize residual tumors is limited. Because of tumor-independent enrichment of nontargeted probes, precise discrimination between tumor and healthy tissue is not possible. In the case of dual-modality indocyanine green- ${ }^{99 \mathrm{~m}} \mathrm{Tc}$-nanocolloids, a distribution along the lymphatic 
system enables the detection of sentinel lymph nodes in the lymphatic drainage area of the primary tumor. Because of the regional application, a high enrichment close to the injection side enhances background uptake in the pelvic area, and lesions outside the lymphatic distribution route might not be identified (7).

To overcome these restrictions, dual-labeled probes specifically targeting prostate cancer cells have been developed. The prostatespecific membrane antigen (PSMA) has been found to be overexpressed in prostate cancer (9). Because the upregulation of PSMA correlates with the malignancy of the carcinoma (10), PSMA became an established target for the diagnosis and therapy of prostate cancer. Besides monomodality probes providing either radionuclide (11-16) or fluorescence imaging (17-20), only a few antibody- or small-molecule-based dual-modality agents targeting the extracellular domain of PSMA have been designed $(21,22)$. However, in antibody approaches, specific tumor visualization is hampered by the long circulation in the blood pool, whereas small-molecule probes show fast pharmacokinetics resulting in clear imaging contrast at early time points after injection. Because the biologic properties of small molecules are typically affected by their conjugation with chelators or fluorescent dyes, the challenge in the design of dual-modality probes is to preserve favorable pharmacokinetic properties along with a high and specific tumor uptake.

In this study, the clinically established $N, N^{\prime}$-bis[2-hydroxy5-(carboxyethyl)benzyl] ethylenediamine- $N, N^{\prime}$-diacetic acid (HBEDCC)-based PET tracer ${ }^{68} \mathrm{Ga}$-Glu-urea-Lys(Ahx)-HBED-CC ( ${ }^{68}$ Ga-PSMA-11) was selected as a low-molecular-weight PSMA inhibitor core structure showing fast clearance from blood and background organs and a high specific uptake in prostate cancer lesions (12, 23,24). Here, we present the preclinical evaluation of novel duallabeled PSMA inhibitors based on PSMA-11 with advantageous pharmacokinetics and considerably improved targeting properties for sensitive pre-, intra-, and postoperative detection of prostate cancer.

\section{MATERIALS AND METHODS}

\section{Chemical Synthesis and Radiolabeling}

All commercially available chemicals, reagents, and solvents were of analytic grade and used without further purification. The bistetrafluorophenyl-functionalized HBED-CC was synthesized as described previously (25). The product with the tetrafluorophenyl-activated sites $(0.157 \mathrm{mmol})$ was reacted with tri-tert-butyl-protected Glu-urea-Lys (0.9 equivalents, $0.141 \mathrm{mmol}, 68.90 \mathrm{mg}$; $\mathrm{ABX}$ ) in dimethylformamide in the presence of $25 \mu \mathrm{L}$ of $N, N$-diisopropylethylamine. After $4 \mathrm{~h}$ at room temperature, an excess of $100 \mu \mathrm{L}$ of $2,2^{\prime}$-(ethylenedioxy)bis(ethylamine) was added, and the mixture was reacted for $16 \mathrm{~h}$ at room temperature to form tri-tert-butyl-protected Glu-urea-Lys[Fe(HBED-CC)]-PEG $-\mathrm{NH}_{2}$. The activated dyes fluorescein isothiocyanate (isomer I, 1 equivalent, $1 \mathrm{mg}$; Merck) and AlexaFluor488 tetrafluorophenyl ester (1 equivalent, $1 \mathrm{mg}$; Thermo Fisher Scientific) were dissolved in $50 \mu \mathrm{L}$ of dimethyl sulfoxide and conjugated to Glu-urea-Lys[Fe(HBED-CC)]-PEG $-\mathrm{NH}_{2}$ (3 equivalents) in dimethylformamide $(300 \mu \mathrm{L})$ and $N, N$-diisopropylethylamine $(15 \mu \mathrm{L})$ for $24 \mathrm{~h}$ at room temperature. The conjugation of IRDye800CW-NHS-ester (1 equivalent, $1 \mathrm{mg}$; LI-COR Biosciences) and Dylight800-NHS-ester (1 equivalent, $1 \mathrm{mg}$; Thermo Fisher Scientific) to Glu-urea-Lys[Fe(HBED-CC)]- $\mathrm{PEG}_{2}-\mathrm{NH}_{2}$ was performed in phosphate-buffered saline (PBS) $(\mathrm{pH} 8.5,300 \mu \mathrm{L})$ for $24 \mathrm{~h}$ at room temperature. Complexed $\mathrm{Fe}^{3+}$ was removed on a Sep-Pack C18 cartridge (Waters) with $1 \mathrm{M} \mathrm{HCl}$ as described previously (25). The final conjugates were purified and analyzed by reversed-phased highperformance liquid chromatography (HPLC) and matrix-assisted laser desorption/ionization mass spectrometry (Table 1). To further
TABLE 1

Analytic Data of Compounds

\begin{tabular}{cccc}
\hline \multicolumn{1}{c}{ Compound } & \multicolumn{2}{c}{$\mathrm{m} / \mathrm{z}$} & $\begin{array}{c}\text { Chemical } \\
\text { yield }(\%)\end{array}$ \\
\cline { 2 - 3 } & Calculated & Found & \\
\hline $\begin{array}{c}\text { Glu-urea-Lys-HBED-CC- } \\
\text { FITC }\end{array}$ & $1,355.4$ & $1,356.9$ & 23 \\
$\begin{array}{c}\text { Glu-urea-Lys-HBED-CC- } \\
\text { AlexaFluor488 }\end{array}$ & $1,478.4$ & $1,481.5$ & 27 \\
$\begin{array}{c}\text { Glu-urea-Lys-HBED-CC- } \\
\text { IRDye800CW }\end{array}$ & $1,950.2$ & $1,951.4$ & 35 \\
$\begin{array}{c}\text { Glu-urea-Lys-HBED-CC- } \\
\text { DyLight800 }\end{array}$ & $1,902.1$ & $1,901.9$ & 18 \\
\hline
\end{tabular}

$\mathrm{m} / \mathrm{z}=$ mass-to-charge ratio $(\mathrm{g} / \mathrm{mol})$ on mass spectrometry of nonlabeled ligand; FITC = fluorescein isothiocyanate.

Chemical yield refers to fluorescent dye conjugation reaction to Glu-urea-Lys[Fe(HBED-CC)]-PEG $2-\mathrm{NH}_{2}$.

characterize the precursor Glu-urea-Lys[Fe(HBED-CC)]- $\mathrm{PEG}_{2}-\mathrm{NH}_{2}$, high-resolution mass spectrometry and nuclear magnetic resonance were additionally performed. Preparative HPLC was performed with LaPrep P110 (VWR) equipped with a Nucleodur Sphinx RP column (VWR) and a variable ultraviolet detector (P314; VWR) with a flow rate of $20 \mathrm{~mL} / \mathrm{min}$. An Agilent 1100 series system (Agilent Technologies) was equipped with $100 \times 10 \mathrm{~mm}$ Chromolith RP-18e columns (Merck) for semipreparative HPLC and with $100 \times 4.6 \mathrm{~mm}$ Chromolith RP-18e columns for analytic HPLC. Ultraviolet absorbance was measured at 214 and $254 \mathrm{~nm}$, respectively.

${ }^{68} \mathrm{Ga}$ (half-life, $68 \mathrm{~min} ; \beta^{+}, 89 \% ; \beta^{+}$maximum energy, $1.9 \mathrm{MeV}$ ) was obtained from a pyrogallol resin support-based ${ }^{68} \mathrm{Ge} /{ }^{68} \mathrm{Ga}$ generator (26). For ${ }^{68} \mathrm{Ga}$ labeling, the precursor peptides (1 nmol in 2-[4(2-hydroxyethyl)piperazin-1-yl] ethanesulfonic acid buffer [580 mg/mL] with $5 \mathrm{mg}$ of ascorbic acid, $90 \mu \mathrm{L}$ ) were added to $40 \mu \mathrm{L}$ of ${ }^{68} \mathrm{Ga}^{-\mathrm{Ga}^{3+}}$ eluate ( $\sim 40 \mathrm{MBq})$. The $\mathrm{pH}$ was adjusted to 3.8 using $\mathrm{NaOH}$; typically, 1-2 $\mu \mathrm{L}$ of $30 \% \mathrm{NaOH}$ were sufficient. The reaction mixture was incubated at $98^{\circ} \mathrm{C}$ for $10 \mathrm{~min}$. The radiochemical yield was determined by analytic reversed-phase HPLC and reversed-phase thin-layer chromatography on silica gel plates (60 RP-18 $\mathrm{F}_{254 \mathrm{~s}}$; Merck) with $0.1 \mathrm{M}$ sodium citrate as a mobile phase. For ${ }^{69 / 71} \mathrm{Ga}$-complexation, the precursor peptides (1 mM in 0.1 M 2-[4-(2-hydroxyethyl)piperazin-1-yl]ethanesulfonic acid buffer, $\mathrm{pH} 7.5,40 \mu \mathrm{L}, 40 \mathrm{nmol}$ ) were reacted with a 10 -fold molar excess of $\mathrm{Ga}(\mathrm{III})$-nitrate (Sigma-Aldrich) in $0.1 \mathrm{~N} \mathrm{HCl}$ $(10 \mu \mathrm{L})$ and $10 \mu \mathrm{L}$ of 2-[4-(2-hydroxyethyl)piperazin-1-yl]ethanesulfonic acid solution $(2.1 \mathrm{M})$ overnight at room temperature. The $\mathrm{pH}$ of the labeling solution was adjusted to 4.2 using $\mathrm{NaOH}$. The lipophilicity of the ${ }^{68} \mathrm{Ga}$-labeled compounds was determined using the 2-phase system $n$-octanol and PBS.

\section{Cell Culturing}

PSMA-positive LNCaP cells (CRL-1740; ATCC) and PSMAnegative PC3 cells (CRL-1435; ATCC) were cultured in RPMI medium enriched with $10 \%$ fetal calf serum and $2 \mathrm{mM}$ L-glutamine (all from PAA). Cells were grown at $37^{\circ} \mathrm{C}$ in humidified air with $5 \% \mathrm{CO}_{2}$ and were harvested using $0.25 \%$ trypsin and $0.02 \%$ ethylenediaminetetraacetic acid (Invitrogen).

\section{Cell Binding and Internalization}

The competitive cell binding assay and internalization experiments were performed as described previously (12). Briefly, competitive 
binding was done by incubating various concentrations of ${ }^{69 / 71} \mathrm{Ga}-$ labeled or noncomplexed compound in the presence of ${ }^{68} \mathrm{Ga}$-labeled radioligand [Glu-urea-Lys(Ahx) $]_{2}$-HBED-CC (PSMA-10; ABX). Internalization was determined by incubation of $30 \mathrm{nM}$ radiolabeled compound for $45 \mathrm{~min}$ at $37^{\circ} \mathrm{C}$ and at $4^{\circ} \mathrm{C}$, respectively. For blocking studies, $500 \mu \mathrm{M}$ 2-(phosphonomethyl)-pentanedioic acid was used. Cell-bound radioactivity and the collected fractions were measured using a $\gamma$-counter (Packard Cobra II; GMI).

\section{Microscopy}

For confocal microscopy, $10^{5}$ cells were seeded in a poly-L-lysinecoated 4-well Nunc Lab-Tek II Chamber Slide system (Thermo Fisher Scientific) $48 \mathrm{~h}$ before incubation. After 1 washing with PBS, the cells were incubated with $300 \mu \mathrm{L}$ of noncomplexed Glu-urea-Lys-HBEDCC-AlexaFluor488 $(10 \mathrm{nM})$ in medium supplemented with $10 \%$ fetal calf serum and $2 \mathrm{mM}$ L-glutamine (all from PAA) for 5, 15, or $30 \mathrm{~min}$, respectively. Blocking studies were performed by incubating a $10 \mathrm{nM}$ solution of noncomplexed Glu-urea-Lys-HBED-CC-AlexaFluor488 for $30 \mathrm{~min}$ in the presence of 2-(phosphonomethyl)-pentanedioic acid $(500 \mu \mathrm{M})$. After incubation, the cells were washed with PBS and fixed for 10 min with paraformaldehyde (2\% in PBS). After 3 washings with PBS, the chamber was removed. For mounting, 1 drop of ProLong Diamond Antifade Mountant with 4',6-diamidino-2-phenylindole dihydrochloride (Thermo Fisher Scientific) per well was used, and the slides were covered with a Karl Hecht cover glass $0.17 \pm 0.01$ $\mathrm{mm}$ thick. The cellular uptake and internalization of the noncomplexed Glu-urea-Lys-HBED-CC-AlexaFluor488 was measured using a confocal Leica TCS SP5 II microscope (Leica) equipped with a $\times 63 / 1.40$ oil objective. The measurement was performed with a 405-nm-diode and Argon laser (488 nm), with detection windows of 415-484 nm for 4',6-diamidino-2-phenylindole dihydrochloride and 498-587 nm for Glu-urea-Lys-HBED-CC-AlexaFluor488.

\section{Biodistribution and Imaging Studies}

For the experimental mouse tumor models, $5 \times 10^{6} \mathrm{LNCaP}$ or PC 3 cells (in 50\% Matrigel; Becton Dickinson) were subcutaneously inoculated into the right trunk of 7- to 8-wk-old male BALB/c nu/nu mice (Charles River Laboratories). The tumors were allowed to grow to a size of approximately $1 \mathrm{~cm}^{3}$. For organ distribution studies, the ${ }^{68} \mathrm{Ga}$-labeled compounds were injected into a tail vein $(1-2 \mathrm{MBq}$, $60 \mathrm{pmol} ; n=3$ ). The animals were sacrificed at 1,2 , or $6 \mathrm{~h}$ after injection. Organs of interest were dissected, blotted dry, and weighed. The radioactivity was measured using a $\gamma$-counter and calculated as $\% \mathrm{ID} / \mathrm{g}$.
For the small-animal PET studies, the mice were anesthetized (2\% sevoflurane; Abbott) and placed into a PET scanner (Inveon PET; Siemens). After a $10-\mathrm{min}$ transmission scan, $0.5 \mathrm{nmol}$ of the ${ }^{68} \mathrm{Ga}$ labeled compounds in $0.9 \% \mathrm{NaCl}(\mathrm{pH} 7)$ were injected into the tail vein, after which a dynamic scan was obtained for $60 \mathrm{~min}$ and a static scan was obtained from 120 to $140 \min (n=1)$. The images were iteratively reconstructed (16 subsets, 4 iterations) applying median root prior correction and were converted to SUV images.

For the small-animal proof-of-concept study, LNCaP tumor-bearing mice underwent small-animal PET imaging for $2 \mathrm{~h}$ after injection of $0.5 \mathrm{nmol}$ of ${ }^{68} \mathrm{Ga}$-Glu-urea-Lys-HBED-CC-IRDye800CW. Afterward, tumors and PSMA-expressing tissue were detected and resected by fluorescence guidance using an IMAGE1 S system (excitation wavelength $805 \mathrm{~nm}$; Karl Storz GmbH and Co. KG).

In a further proof-of-concept study, healthy pigs (2-3 mo old, $n=$ 2) were anesthetized and, after a preinjection imaging acquisition with a da Vinci Firefly system (excitation wavelength $805 \mathrm{~nm}$; Intuitive Surgical, Inc.), received an intravenous injection of $1 \mathrm{mg}$ of noncomplexed Glu-urea-Lys-HBED-CC-IRDye800CW $(30 \mu \mathrm{g} / \mathrm{kg}$ in sterile PBS). One hour after injection, the fluorescence signal was detected and fluorescence-guided prostatectomy, including ex vivo fluorescence control, was performed. All animal experiments complied with the current laws of the Federal Republic of Germany and were conducted according to German Animal Welfare guidelines.

\section{Statistical Aspects}

Experiments were performed at least in triplicate. Quantitative data were expressed as mean $\pm \mathrm{SD}$. If applicable, means were compared using the Student $t$ test (GraphPad Prism, version 7). $P$ values of less than 0.05 were considered statistically significant.

\section{RESULTS}

\section{In Vitro Characterization}

The final products could be identified using reversed-phase HPLC/matrix-assisted laser desorption/ionization mass spectrometry and Glu-urea-Lys[Fe(HBED-CC)]-PEG $2-\mathrm{NH}_{2}$ because the PSMA11-derived precursor proved to be fully consistent with the assigned structure by high-resolution mass spectrometry and nuclear magnetic resonance (Supplemental Figs. 1-10; supplemental materials are available at http://jnm.snmjournals.org). To investigate the influence of the fluorescent dye conjugation to the PSMA-11-derived precursor, internalization efficiency and PSMA-binding affinity were determined (Table 2). The ${ }^{68} \mathrm{Ga}$ complexation of all investigated

TABLE 2

Cell Binding and Internalization of Dye Conjugates in Comparison to PSMA-11 $(25,27)$

\begin{tabular}{|c|c|c|c|c|}
\hline \multirow[b]{2}{*}{ Compound } & \multicolumn{2}{|c|}{${ }^{68} \mathrm{Ga}-$ labeled compounds } & \multicolumn{2}{|c|}{$\mathrm{IC}_{50}(\mathrm{nM})$} \\
\hline & $\begin{array}{c}\text { Specifically cell } \\
\text { surface-bound (\%|A) }\end{array}$ & $\begin{array}{c}\text { Specifically } \\
\text { internalized }(\% \mid A)\end{array}$ & $\begin{array}{l}\text { Metal-free } \\
\text { ligands }\end{array}$ & $\begin{array}{l}\text { 69/71 Ga-labeled } \\
\text { compounds }\end{array}$ \\
\hline PSMA-11 & $18.31 \pm 0.71$ & $3.54 \pm 0.28$ & $19.19 \pm 7.42$ & $12.1 \pm 2.1$ \\
\hline Glu-urea-Lys-HBED-CC-FITC & $1.28 \pm 0.20$ & $4.89 \pm 0.93$ & $11.14 \pm 1.16$ & $10.52 \pm 1.47$ \\
\hline Glu-urea-Lys-HBED-CC-AlexaFluor488 & $5.22 \pm 0.25$ & $14.96 \pm 0.81$ & $13.32 \pm 0.83$ & $25.59 \pm 3.93$ \\
\hline Glu-urea-Lys-HBED-CC-IRDye800CW & $13.62 \pm 4.79$ & $18.70 \pm 7.03$ & $24.54 \pm 5.70$ & $35.54 \pm 2.94$ \\
\hline Glu-urea-Lys-HBED-CC-DyLight800 & $10.50 \pm 3.64$ & $13.88 \pm 7.04$ & $21.41 \pm 1.90$ & $21.23 \pm 4.26$ \\
\hline
\end{tabular}

$\% \mathrm{IA}=$ percentage of applied radioactivity bound to $10^{5}$ cells (specific cell uptake was determined by blockage using $500 \mu \mathrm{M}$ 2-(phosphonomethyl)-pentanedioic acid); FITC = fluorescein isothiocyanate.

Data are mean $\pm \operatorname{SD}(n=3)$. 


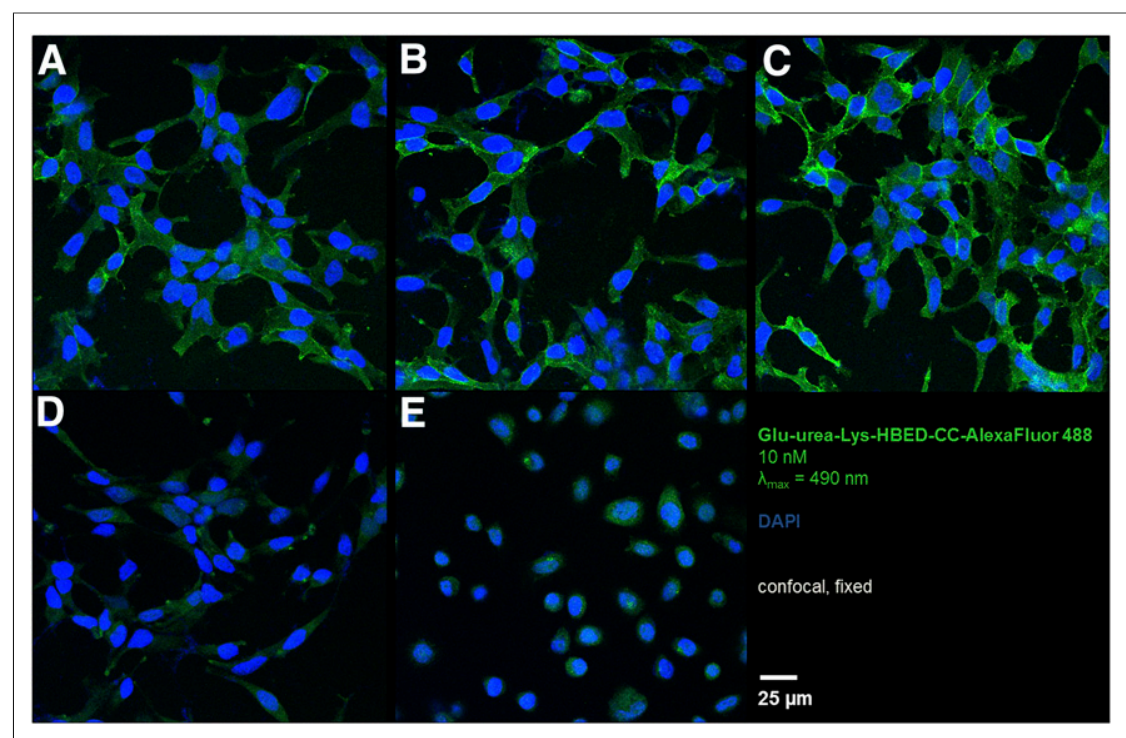

FIGURE 1. Specific cell binding and internalization in confocal microscopy. (A-C) LNCaP cells were incubated with $10 \mathrm{nM}$ noncomplexed Glu-urea-Lys-HBED-CC-AlexaFluor488 for $5 \mathrm{~min}(\mathrm{~A})$, $15 \mathrm{~min}(\mathrm{~B})$, and $30 \mathrm{~min}$ (C) at room temperature and fixed with paraformaldehyde. (D) For blocking studies, 2-(phosphonomethyl)-pentanedioic acid was added and simultaneously incubated for $30 \mathrm{~min}$. PC3 cells as negative control were incubated with $10 \mathrm{nM}$ noncomplexed ligand for $30 \mathrm{~min}$ at room temperature $(\mathrm{E})$. DAPI $=4$ ',6-diamidino-2-phenylindole dihydrochloride; $\lambda_{\max }=$ wavelength of maximum absorbance.

compounds resulted in radiochemical yields higher than $99 \%$. The lipophilicity of the ${ }^{68} \mathrm{Ga}$-labeled compounds was investigated by $\log$ $\mathrm{D}$ determination in comparison to ${ }^{68} \mathrm{Ga}-\mathrm{PSMA}-11(-2.91 \pm 0.06)$ and ranged from -2.01 to -2.98 (Supplemental Table 1).

All dye conjugates showed a high, complexation-independent PSMA-binding affinity in the same nanomolar range as the reference PSMA-11 $(25,27)$. In contrast to the slightly reduced specific cell surface binding, the specific internalization was improved for the ${ }^{68} \mathrm{Ga}$ - and dye-labeled compounds, with a strong, significant enhancement for ${ }^{68} \mathrm{Ga}$-Glu-urea-Lys-HBED-CC-IRDye $800 \mathrm{CW}$ by a factor of $3.9(P<0.05)$. Table 3) (10).
Specific cell binding and internalization could also be visualized by confocal microscopy (Fig. 1). The evaluated compound Glu-urea-Lys-HBED-CC-AlexaFluor488 (noncomplexed) showed an increased binding and intracellular fraction for PSMApositive LNCaP cells over time, whereas for PSMA-negative PC3 cells almost no uptake was detected. Simultaneous incubation with 2-(phosphonomethyl)-pentanedioic acid, a highly specific PSMA inhibitor, resulted in successful blocking of Glu-ureaLys-HBED-CC-AlexaFluor488 uptake.

\section{In Vivo Characterization}

Besides a high PSMA-binding affinity and excellent internalization properties, dye conjugation to the PSMA-11-derived core structure resulted in a high tumor uptake in PSMA-expressing tumors (Fig. 2A; Supplemental Table 3). Compared with the reference ${ }^{68} \mathrm{Ga}-\mathrm{PSMA}-11(4.89 \pm 1.34$ percentage injected dose [\%ID]/g) (27), tumor uptake was significantly increased for the ${ }^{68} \mathrm{Ga}$-labeled conjugates with fluorescein isothiocyanate $(10.86 \pm 0.94 \% \mathrm{ID} / \mathrm{g}$, factor of $2.2, P<0.05)$, IRDye $800 \mathrm{CW}$ $(13.66 \pm 3.73 \% \mathrm{ID} / \mathrm{g}$, factor of $2.8, P<$ $0.05)$, and DyLight800 (15.62 $\pm 5.52 \% \mathrm{ID} / \mathrm{g}$, factor of 3.2 , $P<0.05)$, whereas ${ }^{68}$ Ga-Glu-urea-Lys-HBED-CC-AlexaFluor488 showed a similar tumor uptake $(9.12 \pm 5.47 \% \mathrm{ID} / \mathrm{g}$, factor of $1.9, P>0.05)$. The tumor uptake was proved to be specific by the strongly reduced uptake in PSMA-negative PC3 tumors obtained for all conjugates (Supplemental Table 4). Besides a higher tumor uptake after dye conjugation, the pharmacokinetic properties of PSMA-11 were preserved, meaning excellent background clearance and renal excretion for all conjugates. A typical uptake in PSMAexpressing spleen tissue was also observed (Fig. 2A; Supplemental

Because of their near-infrared fluorescence, ${ }^{68} \mathrm{Ga}$-Glu-urea-Lys-HBED-CC-IRDye800CW and ${ }^{68} \mathrm{Ga}$-Glu-urea-Lys-HBED-CC-DyLight 800 are well suited for further clinical translation. Both conjugates have a similar tumor uptake $(P>0.05)$, but tumor-to-organ ratios for relevant background organs appeared to be higher for the IRDye $800 \mathrm{CW}$ conjugate (tumor-to-blood, 4.49; tumor-to-heart, 4.92; tumor-to-lung, 2.44; tumor-to-liver, 4.94; tumorto-muscle, 4.78) than for the DyLight800 conjugate (tumor-to-blood, 2.18; tumor-to-heart, 3.43; tumor-to-lung, 1.90; tumor-to-liver, 2.54; tumor-to-muscle, 4.06) (Supplemental Table 5).

An additional organ distribution study over different time points was performed with the candidate for clinical translation, ${ }^{68} \mathrm{Ga}-\mathrm{Glu}-$ urea-Lys-HBED-CC-IRDye800CW (Fig. 2B; Supplemental Table 6). After a significant reduction $2 \mathrm{~h}$ after injection (7.94 \pm 0.19 $\% \mathrm{ID} / \mathrm{g}, P<0.05)$, the tumor uptake was 
only slightly decreased between 2 and $6 \mathrm{~h}$ after injection $(6.57 \pm$ $0.63 \% \mathrm{ID} / \mathrm{g}, P<0.05)$, suggesting a stabilization of tumor uptake at later time points. Particularly, tumor-to-organ ratios for background organs were strongly increased over time, indicating that the unspecific uptake was reduced to a minimum after $6 \mathrm{~h}$ (tumor-toblood, 15.56; tumor-to-heart, 12.87; tumor-to-lung, 6.88; tumor-toliver, 6.43; tumor-to-muscle, 12.85) (Supplemental Table 7).

Small-animal PET studies confirmed the findings from the organ distribution study, showing clear visualization of the PSMApositive tumor and fast clearance from background organs for all conjugates, comparable to ${ }^{68}$ Ga-PSMA-11 (Fig. 3 and Supplemental Fig. 11) (12). In PSMA-negative PC3 tumors, ${ }^{68} \mathrm{Ga}$-labeled dye conjugate enrichment in tumor tissue was not observed, proving the high PSMA specificity of the examined conjugates.

\section{Proof-of-Concept Studies}

Besides the successful visualization of PSMA-positive tumors in small-animal PET images, a PSMA-specific fluorescence signal in the tumor tissue could be detected ex vivo (Fig. 4A and Supplemental Fig. 12), whereas background organs (e.g., muscle) appeared gray in the fluorescence mode using ${ }^{68} \mathrm{Ga}$-Glu-urea-Lys-HBED-CCIRDye800CW. A fluorescence signal in kidney and spleen was also measured, as these organs have a physiologic PSMA expression (10). As an additional setting closer to that of clinical surgery, studies on healthy pigs were performed with the robotic da Vinci surgical system (Intuitive Surgical, Inc.) after injection of noncomplexed Glu-urea-Lys-HBED-CC-IRDye800CW (Fig. 4B). One hour after injection, a PSMA-specific fluorescence signal was observed in the prostate expressing PSMA on a physiologic level, whereas surrounding background tissue appeared gray in the surgeon's console. After prostatectomy under fluorescence guidance, fluorescence was confirmed ex vivo, showing a signal in prostate tissue and no signal in surrounding soft tissue (Supplemental Fig. 13).

\section{DISCUSSION}

In the treatment of prostate cancer, precise resection of primary or metastatic tumors has a strong influence on patient outcome. New approaches comprising dual-labeled probes support the surgeon by providing for more simplified preoperative planning through PET/CT or PET/MRI combined with precise intraoperative navigation assistance. This dual-modality technology unites localization of tumor foci through the radioactive signal before surgery with intraoperative radioguidance and highly accurate fluorescence-guided comprehensive resection of tumor tissue.

The feasibility of implementing such a treatment regimen in a clinical work flow was previously demonstrated by van der Poel et al. (8), who showed that multimodal indocyanine green-99m Tc-nanocolloids can visualize sentinel lymph nodes. This rather unspecific approach can be overcome by a targeted probe design $(21,22)$. Because of their favorable pharmacokinetic propertiesparticularly, their fast clearance from the blood pool accompanied by high tumor-tobackground contrast at early time pointslow-molecular-weight PSMA inhibitors provide an optimal basis for the design of dual-modality probes. PSMA-11 was identified to be the ideal core molecule, with successful implementation in the clinical working routine as a PET tracer for the imaging of prostate cancer $(1,23,24)$. The high clinical impact of PSMA-11 on treatment regimens is emphasized by recent studies reporting a change in management after ${ }^{68}$ Ga-PSMA-11 PET in approximately $50 \%$ of patients (28). Further
FIGURE 3. Maximum-intensity projections derived from small-animal PET imaging of athymic nude mice bearing LNCaP and PC3 tumors in right trunk. Scans were evaluated 60 and $120 \mathrm{~min}$ after injection (p.i.) of $0.5 \mathrm{nmol}$ of ${ }^{68} \mathrm{Ga}$-labeled ( $\sim 50 \mathrm{MBq}$ ) Glu-urea-Lys-HBED-CC-fluorescein isothiocyanate (A), Glu-urea-Lys-HBED-CC-AlexaFluor488 (B), Glu-urea-Lys-HBED-CC-IRDye800CW (C), and Glu-urea-Lys-HBED-CC-DyLight800 (D). SUV bw $_{\text {, }}$ SUV normalized for body weight. 


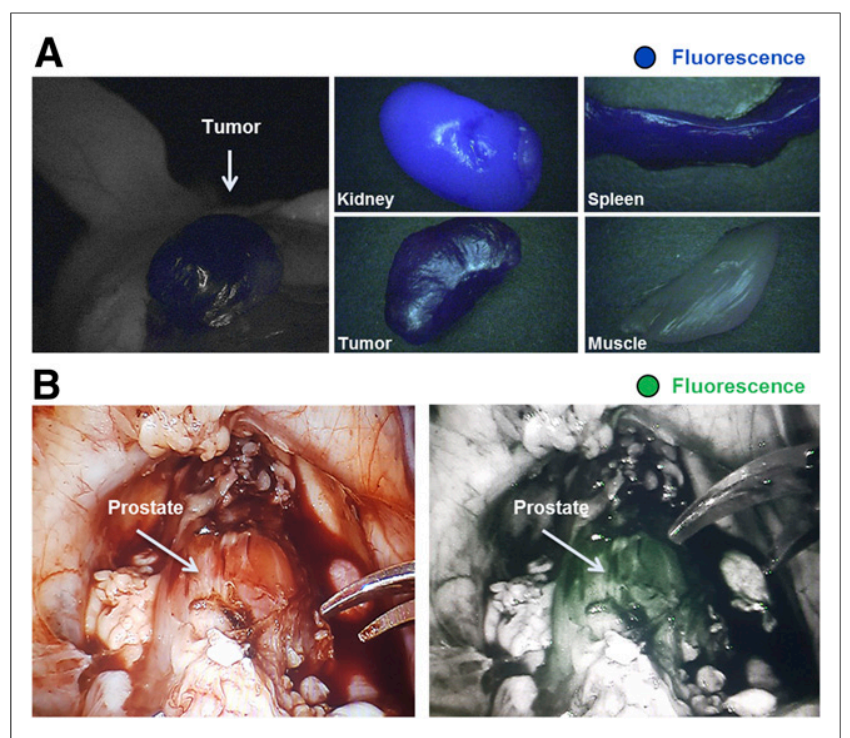

FIGURE 4. Proof-of-concept fluorescence-guided surgery studies in tumor-bearing mice and healthy pigs. (A) ${ }^{68} \mathrm{Ga}-\mathrm{Glu}-$ urea-Lys-HBEDCC-IRDye800CW $(0.5 \mathrm{nmol})$ was injected in mice (LNCaP tumor xenograft) for small-animal PET imaging, followed by ex vivo fluorescence detection after 120 min (IMAGE1 S system). (B) In healthy pigs, after preimaging acquisition of background fluorescence with da Vinci FireFly system, Glu-urea-Lys-HBED-CC-IRDye800CW (30 $\mu \mathrm{g} / \mathrm{kg}$ ) was intravenously injected. Fluorescence-guided prostatectomy accompanied by in vivo and ex vivo fluorescence detection was performed $1 \mathrm{~h}$ after injection.

developed by additional conjugation of a fluorescent dye, dual-labeled PSMA ligands derived from PSMA-11 might considerably improve prostate cancer therapy by precisely guiding the surgeon to tumors. The ${ }^{68} \mathrm{Ga}$-labeled fluorescent dye conjugates presented in this study exhibit high PSMA-binding affinity and strongly increased internalization efficiency. These excellent targeting characteristics improved specific tumor uptake in PSMA-positive lesions by a factor of 3.2 over ${ }^{68} \mathrm{Ga}$-PSMA-11, with high tumor-to-background contrast as early as $1 \mathrm{~h}$ after injection. This improvement might be attributable to the introduction of aromatic groups through fluorescent dyes, potentially further enhancing the interaction within the PSMA-binding pocket, as lipophilic and aromatic moieties have previously been reported to be advantageous (29). The dyes represent lipophilic structures, preferably located distal to the glutamic acid moiety for improved inhibitory potency (30). Because features such as polarity, size, charge, and flexibility also have to be considered, the diverse coupled dyes consequently influence binding properties slightly differently. No correlation between overall lipophilicity and tumor uptake has been observed. The higher tumor uptake might also be attributable to the longer circulation in the blood, which typically also increases the tumor accumulation of biomolecules.

With a strong focus on clinical translation, Glu-urea-Lys-HBEDCC-IRDye800CW and Glu-urea-Lys-HBED-CC-DyLight800 were considered for further proof-of-concept studies because they allow fluorescence guidance in the near-infrared part of the light spectrum. This part of the spectrum has proved ideal for intraoperative fluorescence detection, because near-infrared light can be detected to a depth of about $1 \mathrm{~cm}$ underneath blood and tissue. In addition, this approach is supported by clinically established instruments such as the da Vinci surgical system $(7,8)$. Radioguidance can optionally be performed with this dual-labeled compound in the case of tumors deeper in the pelvis—-beyond the fluorescence detection limit—as the chelator
HBED-CC also allows labeling with radionuclides suitable for radioguidance. Recently published clinical protocols for specific radioguidance provide a proof of concept with this modality (5).

Interestingly, despite comparably high tumor uptake of both conjugates, ${ }^{68} \mathrm{Ga}-\mathrm{Glu}$-urea-Lys-HBED-CC-IRDye800CW showed a higher specificity emphasized by excellent tumor-to-background ratios at $1 \mathrm{~h}$ after injection, with the values even increasing for up to 6 h. Because of the slightly enhanced background activity of ${ }^{68} \mathrm{Ga}-$ Glu-urea-Lys-HBED-CC-DyLight800, which might be attributable to the chemical characteristics of the dye, further in vivo proof-ofconcept studies were conducted with ${ }^{68} \mathrm{Ga}$-Glu-urea-Lys-HBEDCC-IRDye800CW. These studies demonstrated the feasibility of ${ }^{68} \mathrm{Ga}$-Glu-urea-Lys-HBED-CC-IRDye800CW as a small-moleculebased dual-modality probe. In small-animal PET studies, tumors were clearly visualized with high contrast. A specific fluorescence signal could subsequently be detected in PSMA-expressing tissue, providing the surgeon with visual information for the exact localization of relevant PSMA-positive malign tissue and its discrimination from healthy surrounding tissue. Compared with the clinically established PET tracer PSMA-11, the considerably increased tumor uptake with remaining high specificity and successful proof-of-concept studies encourage us to focus on future clinical translation.

\section{CONCLUSION}

PSMA-targeting dual-modality probes have the potential to considerably improve the outcome of prostate cancer therapy, providing the surgeon with precious information through the combined radioactive and fluorescence signals. The PSMA-11based dual-labeled compound ${ }^{68} \mathrm{Ga}$-Glu-urea-Lys-HBED-CCIRDye $800 \mathrm{CW}$ is characterized by high and fast PSMA-specific tumor enrichment and rapid background clearance. This approach allows precise preoperative and intraoperative detection of tumors, along with optional postoperative detection. These features are promising for establishing a prostate cancer treatment regimen supported by a dual-labeled modality.

\section{DISCLOSURE}

This study was supported by VIP+ grant VP00130 from the Federal Ministry of Education and Research (BMBF), Germany. No other potential conflict of interest relevant to this article was reported.

\section{ACKNOWLEDGMENTS}

Technical support was kindly provided by Karl Storz GmbH and Co. KG and by Intuitive Surgical, Inc. Biodistribution and smallanimal PET studies were kindly performed by Karin Leotta and Ursula Schierbaum (German Cancer Research Center, Heidelberg, Germany). Confocal microscopy was kindly supported by the Light Microscopy Core Facility of the German Cancer Research Center. We gratefully thank PD Dr. med. Arianeb Mehrabi (University Hospital Heidelberg, Heidelberg, Germany) and his team for supporting the animal studies.

\section{REFERENCES}

1. Perera M, Papa N, Christidis D, et al. Sensitivity, specificity, and predictors of positive ${ }^{68} \mathrm{Ga}$-prostate-specific membrane antigen positron emission tomography in advanced prostate cancer: a systematic review and meta-analysis. Eur Urol. 2016;70:926-937.

2. Weckermann D, Dorn R, Trefz M, Wagner T, Wawroschek F, Harzmann R. Sentinel lymph node dissection for prostate cancer: experience with more than 1,000 patients. J Urol. 2007;177:916-920. 
3. Mattei A, Fuechsel FG, Bhatta Dhar N, et al. The template of the primary lymphatic landing sites of the prostate should be revisited: results of a multimodality mapping study. Eur Urol. 2008;53:118-125.

4. Stephenson AJ, Eggener SE, Hernandez AV, et al. Do margins matter? The influence of positive surgical margins on prostate cancer-specific mortality. Eur Urol. 2014;65:675-680.

5. Maurer T, Weirich G, Schottelius M, et al. Prostate-specific membrane antigenradioguided surgery for metastatic lymph nodes in prostate cancer. Eur Urol. 2015;68:530-534.

6. van den Berg NS, Buckle T, KleinJan GH, van der Poel HG, van Leeuwen FWB. Multispectral fluorescence imaging during robot-assisted laparoscopic sentinel node biopsy: a first step towards a fluorescence-based anatomic roadmap. Eur Urol. 2017;72:110-117.

7. Brouwer OR, van den Berg NS, Matheron HM, et al. A hybrid radioactive and fluorescent tracer for sentinel node biopsy in penile carcinoma as a potential replacement for blue dye. Eur Urol. 2014;65:600-609.

8. van der Poel HG, Buckle T, Brouwer OR, Valdes Olmos RA, van Leeuwen FW. Intraoperative laparoscopic fluorescence guidance to the sentinel lymph node in prostate cancer patients: clinical proof of concept of an integrated functional imaging approach using a multimodal tracer. Eur Urol. 2011;60:826-833.

9. Schülke N, Varlamova OA, Donovan GP, et al. The homodimer of prostatespecific membrane antigen is a functional target for cancer therapy. Proc Natl Acad Sci USA. 2003;100:12590-12595.

10. Wright GL Jr, Haley C, Beckett ML, Schellhammer PF. Expression of prostatespecific membrane antigen in normal, benign, and malignant prostate tissues. Urol Oncol. 1995;1:18-28.

11. Hillier SM, Kern AM, Maresca KP, et al. ${ }^{123}$ I-MIP-1072, a small-molecule inhibitor of prostate-specific membrane antigen, is effective at monitoring tumor response to taxane therapy. J Nucl Med. 2011;52:1087-1093.

12. Eder M, Schafer M, Bauder-Wust U, et al. ${ }^{68}$ Ga-complex lipophilicity and the targeting property of a urea-based PSMA inhibitor for PET imaging. Bioconjug Chem. 2012;23:688-697.

13. Benešova M, Schafer M, Bauder-Wust U, et al. Preclinical evaluation of a tailormade DOTA-conjugated PSMA inhibitor with optimized linker moiety for imaging and endoradiotherapy of prostate cancer. J Nucl Med. 2015;56:914-920.

14. Weineisen M, Simecek J, Schottelius M, Schwaiger M, Wester H-J. Synthesis and preclinical evaluation of DOTAGA-conjugated PSMA ligands for functional imaging and endoradiotherapy of prostate cancer. EJNMMI Res. 2014;4:63.

15. Foss CA, Mease RC, Fan H, et al. Radiolabeled small-molecule ligands for prostate-specific membrane antigen: in vivo imaging in experimental models of prostate cancer. Clin Cancer Res. 2005;11:4022-4028.

16. Smith-Jones PM, Vallabahajosula S, Goldsmith SJ, et al. In vitro characterization of radiolabeled monoclonal antibodies specific for the extracellular domain of prostate-specific membrane antigen. Cancer Res. 2000;60:5237-5243.
17. Chen Y, Dhara S, Banerjee SR, et al. A low molecular weight PSMA-based fluorescent imaging agent for cancer. Biochem Biophys Res Commun. 2009;390:624-629.

18. Liu T, Wu LY, Hopkins MR, Choi JK, Berkman CE. A targeted low molecular weight near-infrared fluorescent probe for prostate cancer. Bioorg Med Chem Lett. 2010;20:7124-7126.

19. Nakajima T, Mitsunaga M, Bander NH, Heston WD, Choyke PL, Kobayashi H. Targeted, activatable, in vivo fluorescence imaging of prostate-specific membrane antigen (PSMA) positive tumors using the quenched humanized J591 antibodyindocyanine green (ICG) conjugate. Bioconjug Chem. 2011;22:1700-1705.

20. Humblet V, Lapidus R, Williams LR, et al. High-affinity near-infrared fluorescent small-molecule contrast agents for in vivo imaging of prostate-specific membrane antigen. Mol Imaging. 2005;4:448-462.

21. Lütje S, Rijpkema M, Franssen GM, et al. Dual-modality image-guided surgery of prostate cancer with a radiolabeled fluorescent anti-PSMA monoclonal antibody. J Nucl Med. 2014;55:995-1001.

22. Banerjee SR, Pullambhatla M, Byun Y, et al. Sequential SPECT and optical imaging of experimental models of prostate cancer with a dual modality inhibitor of the prostate-specific membrane antigen. Angew Chem Int Ed Engl. 2011;50:91679170.

23. Afshar-Oromieh A, Haberkorn U, Eder M, Eisenhut M, Zechmann CM. [ $\left.{ }^{68} \mathrm{Ga}\right]$ gallium-labelled PSMA ligand as superior PET tracer for the diagnosis of prostate cancer: comparison with ${ }^{18}$ F-FECH. Eur J Nucl Med Mol Imaging. 2012;39:10851086.

24. Afshar-Oromieh A, Malcher A, Eder M, et al. PET imaging with a $\left[{ }^{68} \mathrm{Ga}\right]$ galliumlabelled PSMA ligand for the diagnosis of prostate cancer: biodistribution in humans and first evaluation of tumour lesions. Eur J Nucl Med Mol Imaging. 2013;40: 486-495.

25. Schäfer M, Bauder-Wust U, Leotta K, et al. A dimerized urea-based inhibitor of the prostate-specific membrane antigen for ${ }^{68} \mathrm{Ga}$-PET imaging of prostate cancer. EJNMMI Res. 2012;2:23.

26. Schuhmacher J, Maier-Borst W. A new ${ }^{68} \mathrm{Ge} /{ }^{68} \mathrm{Ga}$ radioisotope generator system for production of ${ }^{68} \mathrm{Ga}$ in dilute $\mathrm{HCl}$. Int J Appl Radiat Isot. 1981;32:31-36.

27. Baranski AC, Schafer M, Bauder-Wust U, et al. Improving the imaging contrast of ${ }^{68} \mathrm{Ga}$-PSMA-11 by targeted linker design: charged spacer moieties enhance the pharmacokinetic properties. Bioconjug Chem. 2017;28:2485-2492.

28. Hope TA, Aggarwal R, Chee B, et al. Impact of ${ }^{68}$ Ga PSMA-11 PET on management in patients with biochemically recurrent prostate cancer. $\mathrm{J} \mathrm{Nucl} \mathrm{Med}$. 201;58:1956-1961.

29. Zhang AX, Murelli RP, Barinka C, et al. A remote arene-binding site on prostate specific membrane antigen revealed by antibody-recruiting small molecules. J Am Chem Soc. 2010;132:12711-12716.

30. Maung J, Mallari JP, Girtsman TA, et al. Probing for a hydrophobic a binding register in prostate-specific membrane antigen with phenylalkylphosphonamidates. Bioorg Med Chem. 2004;12:4969-4979. 